\title{
Intermédialités
}

Histoire et théorie des arts, des lettres et des techniques

Intermediality

History and Theory of the Arts, Literature and Technologies

\section{Jeux narratifs, fictions ludiques}

\section{Marie-Laure Ryan}

Numéro 9, printemps 2007

Jouer

Playing

URI : https://id.erudit.org/iderudit/1005527ar

DOI : https://doi.org/10.7202/1005527ar

Aller au sommaire du numéro

\section{Éditeur(s)}

Centre de recherche sur l'intermédialité

ISSN

1705-8546 (imprimé)

1920-3136 (numérique)

Découvrir la revue

Citer cet article

Ryan, M.-L. (2007). Jeux narratifs, fictions ludiques. Intermédialités /

Intermediality, (9), 15-34. https://doi.org/10.7202/1005527ar

\section{Résumé de l'article}

Partant de la définition du jeu de Johan Huizinga et de la taxinomie de Roger Caillois, cet article examine les relations entre le jeu, la fiction et le récit. La dimension ludique de la fiction tient dans le fait qu'elle repose sur un acte de faire semblant. Inversement, un jeu possède une dimension fictionnelle quand son terrain représente un monde et quand les actions du joueur, au lieu de suivre des règles abstraites et conventionnelles, imitent des actions concrètes dont le but répond à des intérêts authentiquement humains. Caillois pensait que la fiction est incompatible avec les jeux de type ludus, basés sur les règles et la compétition, mais les jeux vidéo, ainsi que les diverses tentatives, dès le $\mathrm{XVII}^{\mathrm{e}}$ siècle, d'associer le jeu de l'oie à des thèmes narratifs démentissent son opinion. La réconciliation du jeu et de l'histoire ne se réalisera toutefois pleinement que lorsque deux conditions seront remplies : 1 . le joueur sera motivé par l'intérêt pris à l'histoire; 2. le joueur construira l'histoire par ses actions, et chaque fois qu'il jouera, il produira une nouvelle histoire.
Ce document est protégé par la loi sur le droit d'auteur. L'utilisation des services d’Érudit (y compris la reproduction) est assujettie à sa politique d'utilisation que vous pouvez consulter en ligne.

https://apropos.erudit.org/fr/usagers/politique-dutilisation/ 


\title{
Jeux narratifs, fictions ludiques
}

\author{
Marie-Laure Ryan
}

\begin{abstract}
U niversellement présents dans les sociétés humaines, les jeux et les histoires sont des modes de divertissement si fondamentaux que la tentation est forte d'essayer de doubler le plaisir en «jouant» les histoires, ou en «historiant» les jeux, c'est-à-dire en les dotant d'un intérêt narratif. On parle par ailleurs d'une «fictionnalité » inhérente au jeu, et d'une «ludicité » inhérente à la fiction. C'est cette double affinité du jeu pour la fiction et pour les histoires que je me propose ici d'explorer.
\end{abstract}

\section{LA FICTION COMME JEU, LE JEU COMME FICTION}

Si tout le monde peut intuitivement donner l'exemple d'un jeu (les échecs) et d'une activité non ludique (faire la vaisselle), et peut-être aussi nommer des activités situées à mi-chemin (siffloter, gribouiller), la notion de jeu demeure notoirement difficile à définir. Ludwig Wittgenstein la comparait à la ressemblance entre les membres d'une même famille: il n'y aurait pas de traits communs à tous les jeux, mais un ensemble de propriétés inégalement distribuées entre eux, sans qu'aucune de ces propriétés ne soit ni nécessaire ni partie d'un ensemble suffisant $^{1}$. On pourrait appeler cette idée de ressemblance de famille un ensemble flou décentré. Mais il existe aussi des ensembles flous qui sont organisés autour de prototypes. Dans cette perspective, il y aurait un groupe de traits pertinents à la notion de jeu, dont certains seraient obligatoires et d'autres facultatifs. Les prototypes différeraient des exemples marginaux par le nombre de traits facultatifs

1. Ludwig Wittgenstein, Investigations philosophiques, trad. Pierre Klossowski, Paris, Éditions Gallimard, coll. «Tel», 1995 [1945], \1-32, p. 115-130. 
qu'ils possèdent. Il serait difficile de trouver des jeux plus prototypiques que ceux qui satisfont la définition classique de Johan Huizinga:

Sous l'angle de la forme, on peut donc, en bref, définir le jeu comme une action libre, sentie comme fictive et située en dehors de la vie courante, capable néanmoins d'absorber totalement le joueur; une action dénuée de tout intérêt matériel et de toute utilité; qui s'accomplit en un temps et dans un espace expressément circonscrits, se déroule avec ordre selon des règles données et suscite dans la vie des relations de groupe s'entourant volontiers de mystère ou accentuant par le déguisement leur étrangeté vis-à-vis du monde habituel ${ }^{2}$.

Dans cette définition, le caractère fictif du jeu est mis au rang de propriété fondamentale. Mais que faut-il entendre par ce terme de «fictif», et quelle est sa relation avec la notion plus technique de fictionnalité, telle qu'on l'utilise pour la littérature et certains arts visuels (le cinéma en particulier)? J'ignore quel mot l'auteur emploie dans le texte original allemand, mais dans la traduction anglaise de Meyer Burach, qui s'appuie à la fois sur l'original et sur une traduction anglaise due à Huizinga lui-même, le terme fictif n'apparaît pas. L'action ludique y est caractérisée comme «standing quite consciously outside ordinary life as being "not serious" 3 ». C'est donc sur son manque de sérieux que repose le caractère fictif du jeu. Difficile à cerner (d'autant plus que Huizinga insiste ailleurs sur le sérieux, c'est-à-dire sur la passion que le joueur apporte au jeu), cette notion de manque de sérieux me semble constituer moins une propriété autonome qu'une conséquence des autres conditions mentionnées dans la définition ci-dessus: l'isolement spatial et temporel du jeu par rapport à la vie courante, son absence de conséquences durables pour les joueurs, son caractère facultatif (personne en principe ne devrait être obligé de jouer), et le fait que les besoins psychiques qui motivent les joueurs, pour importants qu'ils soient à la santé mentale, semblent moins urgents que les besoins strictement matériels. On peut se passer de jeu, mais on ne peut pas se passer de manger.

Le manque de sérieux forme également le pivot de certaines définitions de la fictionnalité littéraire, tout particulièrement de celles qui s'inspirent de la théorie des actes de langage. Pour J. L. Austin ${ }^{4}$, le fondateur de cette théorie, l'énoncé d'un acteur qui fait une promesse sur scène est non sérieux car l'acteur n'est pas

2. Johan Huizinga, Homo Ludens : essai sur la fonction sociale du jeu, trad. Cécile Seresia, Paris, Éditions Gallimard, 1951, p. 13 (je souligne).

3. Johan Huizinga, Homo Ludens: A Study of the Play-Element in Culture, Boston, Beacon Press, 1950, p. 13.

4. John Langshaw Austin, How To Do Things With Words, Oxford, Oxford University Press, 1962. 
personnellement tenu de la remplir5 ${ }^{5}$ Austin considère cet emploi du langage comme étiolé ou parasitique par rapport au langage ordinaire. Reprenant l'idée de suspension de la responsabilité du locuteur, John Searle ${ }^{6}$ définit la fiction comme une assertion feinte (ou comme l'imitation d'une assertion) exécutée sans intention trompeuse. Au lieu de nous renseigner sur ses habitudes personnelles, le romancier qui écrit «longtemps je me suis couché de bonne heure» joue le rôle d'un narrateur, et c'est au personnage du narrateur que le lecteur peut attribuer les croyances impliquées par cette déclaration. À l'auteur qui joue le jeu de la fiction avec le lecteur s'oppose ainsi le narrateur qui parle (en général, mais il y a des exceptions) sérieusement au narrataire.

Alors que l'approche de Searle fait de la fiction une feintise qui porte strictement sur les actes de langage, Kendall Walton la conçoit comme un phénomène qui transcende les médias ${ }^{7}$. Approfondissant la similarité entre le jeu et la fiction, il définit cette dernière comme « un accessoire dans un jeu de faire semblant» ( « a prop in a game of make-believe ») dont la fonction est de faire travailler l'imagination, plutôt que d'implanter des croyances. De même que les enfants qui jouent au Grand Méchant Loup adoptent des personnalités imaginaires et font

5. La performance de l'acteur est assurément une fiction, mais son statut de jeu est questionnable. S'inspirant de l'usage courant, qui parle de «jouer un rôle», Caillois le considère comme un jeu à part entière; mais le statut professionnel de l'acteur, et la division entre l'agent et le bénéficiaire de la performance, mettent en doute cette assimilation. Contrairement à l'enfant qui incarne un personnage au cours d'un jeu de faire semblant comme celui du Grand Méchant Loup, l'acteur ne joue pas principalement pour son plaisir, mais pour le divertissement du public. On pourrait considérer la performance théâtrale comme une dégénérescence du jeu de faire semblant, comme le football professionnel est selon Huizinga une dégénérescence du football joué spontanément: «neither play nor earnest» (Johan Huizinga, Homo Ludens, p. 197). Une autre possibilité consisterait à considérer une activité comme un jeu à part entière quand il est possible de s'y livrer par pur plaisir et sans espoir de gain, même si dans certaines parties les participants ne respectent pas ces conditions. Comme le remarque Jesper Juul, «even professional players are playing a game [...] but in this specific game session, the consequences have been negotiated to be financial and career-determining (Jesper Juul, Half Real: Video Games Between Real Rules and Fictional Words, Cambridge, Massachusetts, The MIT Press, p. 36, c'est l'auteur qui souligne). Dans cette perspective, la performance d'une pièce de théâtre serait un jeu de faire semblant renégocié par ses participants. Mais si cette analyse explique ce que font les acteurs, elle ne rend pas compte du rôle du public.

6. John Searle, «The Logical Status of Fictional Discourse», New Literary History, vol. $6, \mathrm{n}^{\circ} 2,1975$, p. 319-332.

7. Kendall Walton, Mimesis as Make-Believe: On the Foundations of the Representational Arts, Cambridge, Massachusetts, Harvard University Press, 1990. 
semblant qu'un certain arbre est la maison du loup, les spectateurs d'un film font semblant d'être les témoins directs des événements représentés sur l'écran, le spectateur d'un tableau qui montre un bateau s'imagine être en présence d'un bateau $^{8}$ et les lecteurs d'un texte de fiction font semblant que le texte représente l'évocation par le narrateur d'un monde qui existe indépendamment du texte, alors qu'ils savent très bien que ce monde est inventé par l'auteur. Dans chacun de ces exemples, la fictionnalité réside dans l'acte de prendre volontairement un objet pour ce qu'il n'est pas : l'arbre devient maison, le comportement des acteurs relayé par l'image cinématographique devient la vie elle-même, la toile devient bateau, et le texte d'imagination devient un texte référentiel et documentaire.

Laissant de côté le cas épineux de la fictionnalité picturale, qui selon Walton concerne l'art visuel tout entier et n'affecte donc pas la manière dont le spectateur interprète une image (car pour avoir des conséquences cognitives, les jugements de fictionnalité doivent faire des distinctions entre les objets d'un même médium), on peut ainsi associer sans problèmes le cinéma d'acteurs par opposition au cinéma documentaire, le théâtre par opposition aux actions pratiques, et la fiction littéraire par opposition aux textes référentiels à la performance d'un rôle, que ce soit celle de l'acteur qui incarne un personnage, ou celle d'un auteur qui revêt le masque d'un narrateur. Comme l'écrit Jean-Marie Schaeffer : «On ne peut pas comprendre ce qu'est la fiction si on ne part pas des mécanismes fondamentaux du "faire comme si" - de la feintise ludique - et de la simulation imaginative dont la genèse s'observe dans les jeux de rôle et les rêveries de la petite enfance ${ }^{9}$. »

Mais si toute fiction est une feintise ludique, et si des jeux comme le Grand Méchant Loup, les gendarmes et les voleurs, ou le papa et la maman sont des fictions au même titre que Madame Bovary (Gustave Flaubert, 1857) ou les films de la série Star Wars (1977-2005) - sans pour autant être des œuvres littéraires, car la fiction transcende la littérature - , il ne s'ensuit pas que tout jeu soit une fiction. Dans Les jeux et les hommes, un ouvrage fondamental pour l'étude des jeux, Roger Caillois distingue les jeux de faire semblant, qu'il appelle mimicry,

8. C'est là la position de Walton, mais elle me semble la plus contestable de ces analogies entre fiction et jeu de faire semblant. Un tableau figuratif, selon son style, peut en effet être pris pour le signe d'un objet, plutôt que suggérer la présence de cet objet. Pour une critique, voir Marie-Laure Ryan, Avatars of Story, Minneapolis, University of Minnesota Press, 2006, chapitre 2, surtout p. 36-37.

9. Jean-Marie Schaeffer, Pourquoi la fiction?, Paris, Éditions du Seuil, «Poétique», 1999, p. 11. 
des jeux de compétition (agôn) et des jeux de hasard $(\text { alea })^{10}$. De ces trois types de jeux, c'est uniquement le premier qui autorise une assimilation du jeu et de la fiction. Dans la mesure où la fiction repose sur le «comme si », elle présuppose une dimension mimétique que n’offrent en général ni les jeux de compétition ni les jeux de hasard. Des événements tels que marquer un but dans une partie de football ou mettre une pièce échec et mat (une pièce dénommée roi, mais qu'on pourrait tout aussi bien baptiser cornichon) sont entièrement déterminés par les règles conventionnelles du jeu, et n'ont pas d'équivalent hors de son espace. Un jeu qui n'imite rien requiert la faculté de résoudre des problèmes, mais il ne nous demande pas de former une représentation mentale d'objets concrets. J'appellerai ici la faculté de résoudre des problèmes «l'imagination tactique », et la représentation mentale d'objets concrets l'«imagination imaginante». Alors que l'imagination tactique s'exerce sur un terrain de jeu, l'imagination imaginante transforme ce terrain en monde, c'est-à-dire en un espace situé dans le temps habité par des entités individualisées et identifiables. Je considère comme fictionnel tout jeu capable de créer un monde, et de faire par conséquent appel non seulement à l'imagination tactique mais aussi à l'imagination imaginante ${ }^{11}$.

10. En fait la typologie de Caillois comprend une quatrième catégorie dont le statut de jeu est plus contestable: ilinx, terme par lequel Caillois décrit les activités qui déstabilisent la perception et provoquent une sensation de vertige et de panique voluptueuse, comme les attractions de foire ou certains sports comme l'alpinisme. Voir Roger Caillois, Les jeux et les hommes. Le masque et le vertige, Paris, Éditions Gallimard, 1958, chapitre 2. Désormais, les références à cet ouvrage seront indiquées par le sigle «JH», suivi de la page, et placées entre parenthèses dans le corps du texte.

11. En distinguant les jeux qui créent un monde fictionnel de ceux dont l'action consiste à manipuler des objets abstraits, je conteste l'idée, avancée par Sébastien Genvo, que tout jeu digne d'être joué donne lieu à ce que Jean-Marie Schaeffer appelle l'immersion fictionnelle. D'un jeu tel que Tetris (version arcade, 1988), le prototype d'un jeu abstrait, Genvo écrit qu'il présente «un fort potentiel d'adaptation à l'attitude ludique du joueur, permettant de ce fait l'adoption d'une immersion fictionnelle» (Sébastien Genvo, «Les conditions de validité de l'immersion vidéoludique: pour une approche descriptive de la jouabilité», L'observatoire des mondes numériques en sciences humaines, http://www.omnsh.org/article.php3?id_article=88, p. 10). À mon avis, il faut faire une distinction entre un type d'immersion qui résulte d'une intense absorption dans une activité quelconque, et un autre type d'immersion qui provient d'un acte d'imagination imaginante. Seul le second peut s'apparenter à une immersion fictionnelle. Les jeux abstraits provoquent le premier type d'immersion exclusivement; les jeux qui créent un monde fictionnel peuvent combiner les deux types. 


\section{DES FICTIONS LUDIQUES AUX JEUX FICTIONNELS}

Tout jeu n'est donc pas une fiction. Inversement, si toute fiction participe de l'esprit ludique par l'acte de faire semblant sur lequel elle se fonde, la parenté entre la fiction et le jeu présente divers degrés. Entre les fictions ordinaires, dont le discours imite un discours sérieux et les jeux fictionnels, qui invitent l'usager à poursuivre un enjeu spécifique par une participation littéralement (c'est-à-dire physiquement) active se situe la catégorie intermédiaire des fictions ludiques, qui sont pénétrées de part en part par l'esprit du jeu mais qui ne donnent pas de tâche précise à l'utilisateur, n'imposent pas un terme à son activité, et ne lui permettent pas de gagner ou de perdre.

Pour saisir ces degrés de parenté, retournons vers Caillois. En plus des types de jeux mentionnés ci-dessus, Caillois propose une opposition entre deux catégories qui recoupent celles d'agôn, d'alea, et de mimicry. «À une extrémité règne, presque sans partage, un principe commun de divertissement, de turbulence, d'improvisation libre et d'épanouissement insouciant, par où se manifeste une certaine fantaisie incontrôlée qu'on peut designer sous le nom de paidia.» (JH, p. 48) À l'extrémité opposée, Caillois situe une composante qu'il appelle ludus, et qu'il définit comme un besoin «de se plier à des conventions arbitraires, impératives et à dessein gênantes » qui rendent malaisé de parvenir au résultat désiré. Ce résultat demeure "parfaitement inutile, quoiqu'il exige une somme constamment accrue d'efforts, de patience, d'adresse ou d'ingéniosité» (JH, p. 48) ${ }^{12}$. Dans l'ensemble flou que sont les jeux, ceux du type ludus sont en général considérés comme plus centraux que ceux du type paidia. Ludus est défini par des règles et par un but précis qui lui donnent une identité stable et le distinguent clairement des non-jeux; paidia par contre renvoie à un esprit ludique qui s'infiltre à divers degrés dans de nombreuses activités.

Cet esprit se manifeste dans les textes littéraires par des procédés (ou fautil dire des trucs?) tels que les jeux de mots, l'ironie, la parodie, le paradoxe, la métalepse (rencontre de l'auteur avec ses personnages; personnages qui pénètrent dans le monde d'un livre), l'autoréférence, l'autocontradiction, la rupture de l'illusion (auteur interpellant le lecteur ou commentant sur la performance du narrateur), les graphismes variés, et les rencontres farfelues de mots dues au

12. Jesper Juul (Half Real, p. 36) a récemment proposé une définition de ce qu'il appelle les jeux prototypiques qu'on peut considérer comme une formalisation du concept de ludus: 1. les jeux prototypiques sont basés sur des règles; 2 . ils ont un résultat variable et quantifiable; 3. une valeur (gagner ou perdre) est attribuée au résultat; 4 . les joueurs mettent de l'effort à atteindre ce résultat; 5. les joueurs sont attachés émotionnellement au résultat - ils veulent gagner et détestent perdre. 
hasard (le jeu des cadavres exquis cher aux Surréalistes). On reconnaîtra dans cette liste les formules de composition favorites de la littérature post-moderne, mais l'esprit ludique se manifeste déjà dans les effets de miroir du Don Quichotte (1605-1615) de Cervantès (le héros lisant le récit de ses propres aventures), dans les intrusions d'auteur des romans de Diderot (se demandant s'il doit rendre cocu le héros de Jacques le fataliste, 1765-1783), et dans les pages blanches, digressions, et autres arabesques narratives du Tristram Shandy (1759-1767) de Laurence Sterne. Si l'espièglerie des fictions ludiques fait d'elles des manifestations exemplaires de paidia, elles ne sont pas nécessairement ennemies des règles, pour autant que ces règles représentent une difficulté librement choisie qui permet à l'auteur de déployer sa virtuosité dans la solution du problème. C'est à un mélange de ludus et de paidia qu'il faut attribuer les formes fixes de la poésie médiévale et les systèmes de contraintes prisés par les auteurs d'Oulipo, systèmes qui produisent des figures telles que les anagrammes, lipogrammes et palindromes.

Alors que les fictions ludiques de la littérature imprimée sont un spectacle offert au lecteur, le vrai joueur étant l'auteur qui exhibe son adresse à vaincre les obstacles ou à jongler avec le langage, leurs sœurs cybernétiques (hypertextes, poésie numérique, et certaines installations artistiques) sont des activités participatives. Comme dans les jeux canoniques, le lecteur de ces fictions intervient activement, soit en cliquant des boutons, soit en fouillant l'écran avec la souris pour déclencher des événements, soit en contrôlant délibérément les objets sur l'écran, mais au lieu de viser un but précis, son activité est une flânerie à travers le texte, une exploration sans autre fin que l'exaltation de la découverte.

Les fictions ludiques tournent vers le jeu fictionnel avec les jeux de faire semblant comme le Grand Méchant Loup ou les jeux vidéo de simulation comme les Sims (Maxis Software/Electronic Arts, 2000) ou SimCity (Maxis Software/ Brøderbund Software, 1989). Rappelons que dans le jeu de simulation, le joueur gère un système (tels qu'une ville ou une famille) dont il contrôle l'évolution en choisissant lui-même ses objectifs, au lieu de poursuive des buts déterminés par les règles du jeu. S'il y a possibilité de perdre, quand le système est si mal géré qu'il devient totalement dysfonctionnel, ou quand l'ordinateur crée des catastrophes qui le détruisent (tremblement de terre pour la ville, incendie pour la famille), il n'y a par contre pas moyen de gagner, puisque le monde fictionnel, comme le monde actuel, est en constante évolution. Représentants exemplaires de la paidia ${ }^{13}$, ces jeux donnent libre cours à l'improvisation et à l'imagination imaginante.

13. Comme le remarque Gonzalo Frasca, «Ludology Meets Narratology: Similitudes and Differences between (Video)Games and Narrative », 1999, http://www.ludology.org/ articles/ludology.htm. 
Est-il possible de combiner fiction et ludus, pour la réalisation la plus complète de l'idée de jeu? Caillois pensait que la fiction était incompatible avec les règles, qui forment la quintessence du ludus: «Ainsi, les jeux ne sont pas réglés et fictifs. Ils sont plutôt ou réglés ou fictifs» (JH, p. 41). La plupart des jeux réglés et compétitifs dans les années 1950, époque où écrivait Caillois, étaient en effet des jeux abstraits, sans équivalent dans la réalité : échecs, go, mots croisés, et les diverses formes de sport (à part ceux qui dérivent d'une activité pratique, comme l'escrime). Mais trente ans après la parution du livre de Caillois, le développement des jeux vidéo semble apporter un démenti à son jugement. Les jeux vidéo d'«aventures » ou de type «shooters » appartiennent clairement au ludus par leur nature compétitive, leur organisation en niveaux à conquérir, et la stricte limitation du champ d'activité du joueur par le code de l'ordinateur.

Au lieu de demander aux joueurs d'accumuler des points en touchant certaines cibles tout en évitant certains obstacles, ces jeux leur assignent toutefois des missions concrètes, telles que protéger la terre d'envahisseurs venus d'une autre planète, ou sauver des princesses kidnappées par des dragons. Les jeux vidéo des quinze dernières années donnent de surcroît au terrain de jeu l'apparence d'un monde aux paysages richement détaillés, et invitent le joueur à s'identifier à un personnage qui possède non seulement une apparence visible, mais dans certains cas des traits de caractère ou des aptitudes individualisés. Les missions du joueur sont "parfaitement inutiles» pour sa vie pratique, ainsi que Caillois définissait les résultats du ludus, mais elles représentent des quêtes lourdes de conséquences du point de vue de l'avatar. Mais si l'imagination imaginante n'est pas radicalement incompatible avec l'imagination stratégique nécessaire pour contourner les obstacles, cela ne veut pas dire que ces deux types d'imagination possèdent le même poids dans la balance du jeu. Comme nous allons le voir, les diverses tentatives de fictionnaliser les jeux en les rattachant à une histoire font face à un problème majeur, celui de trouver un équilibre satisfaisant entre l'histoire et ce qu'on appelle dans le jargon ludologiste la «jouabilité » ("gameplay»), terme qui dénote les possibilités d'action du joueur.

\section{HISTORIER LES JEUX OU JOUER LES HISTOIRES ?}

Il existe deux stratégies majeures pour doter les jeux d'un intérêt narratif: partir d'un ensemble de règles et de buts, et les vêtir d'une histoire pour les rendre plus attrayantes pour le joueur; ou partir d'une histoire, et la transformer en jeu, pour permettre au joueur de s'attarder dans un monde fictionnel familier.

Selon Chris Crawford, la première stratégie est la plus courante dans l'industrie des jeux vidéo ${ }^{14}$. On crée un schéma d'action, des modes de participation,

14. Chris Crawford, Chris Crawford on Interactive Storytelling, Berkeley, New Riders, 2004 , p. 69. 
un type d'interface, ou de nouvelles ressources, comme la caméra qui permet de filmer le jeu, et on fait appel à un scénariste dans les derniers stades du développement pour emballer toutes ces inventions dans une histoire. Mais si la trame narrative aide à vendre le jeu (elle inspire des illustrations sur le coffret qui promettent une action aussi captivante que celle d'un film), elle est vite oubliée quand les joueurs sont immergés dans la partie. L'attrait du genre des shooters à la première personne tels que Doom (id Software/id Software, 1993), Quake (id Software/id Software, 1996) ou Half-Life (Valve/Sierra On-line, 1998), exemples prototypiques de jeux produits par cette méthode, ne réside pas dans l'intérêt de l'intrigue, mais dans la nouveauté des armes, la sensation de danger, la rapidité des réactions du système aux actions du joueur, et le réalisme de l'image, autrement dit, dans l'intensité de la compétition et dans le spectacle de la technologie. Pour le joueur en proie à l'adrénaline du combat, peu importe la cause pour laquelle il se bat.

L'historisation d'un jeu aux règles préétablies n'est pas unique aux jeux vidéo, comme le démontrent les nombreuses tentatives d'associer une intrigue au vénérable jeu de l'oie. Ce jeu, qui date de la Renaissance et tire son nom de l'oiseau qui avertit Rome de l'approche des Gaulois, se joue comme on le sait sur un plateau représentant un labyrinthe monopériple en forme de spirale comprenant le plus souvent 63 cases ornées d'images. L'enjeu est d'être le premier joueur à atteindre le centre du labyrinthe, espace où sont généralement inscrites les règles. Les joueurs progressent vers le but en avançant leur pion d'autant de cases que le nombre jeté par une paire de dés. C'est donc un jeu qui combine l'agôn et l'alea. Pour instiller du suspense dans la partie, certaines cases, souvent marquées par l'image de l'oie, sont associées à des règles néfastes ou favorables au joueur: perdre son tour, avancer d'un certain nombre de cases, ou pour le malheureux joueur qui tombe sur la case 58 , retourner au point de départ. Comme le remarquent Katie Salen et Eric Zimmerman, on pourrait très bien se passer de plateau, de labyrinthe et d'images en formulant les règles de la manière suivante:

1. Tous les joueurs partent avec un total de o.

2. Les joueurs, tour à tour, ajoutent à leur total un nombre tiré au sort.

3. Si le total d'un joueur excède 63 , ne pas ajouter le nombre.

4. Si le total atteint certains nombres, il change. Par exemple, à 15 ajouter 5 ou à 39 retrancher 7 . Pour certains nombres, passer un tour. Si le total est 58 , il devient 0 .

5. Le premier joueur à atteindre un total de 63 gagne $^{15}$.

15. Katie Salen et Eric Zimmerman, Rules of Play: Game Design Fundamentals, Cambridge, Massachusetts, The MIT Press, 2003, p. 133. 
Réduit à cette structure abstraite, le jeu de l'oie a tout l'agrément d'une leçon de calcul. Sa popularité durable ne tient pas dans l'ingénuité des règles, qui ne laissent aucun choix aux joueurs, mais dans les diverses manières de l'habiller de thèmes et d'illustrations qui doublent l'agrément social de la compétition entre amis d'une satisfaction artistique autant qu'imaginative (voir figures 1, 2 et 3).

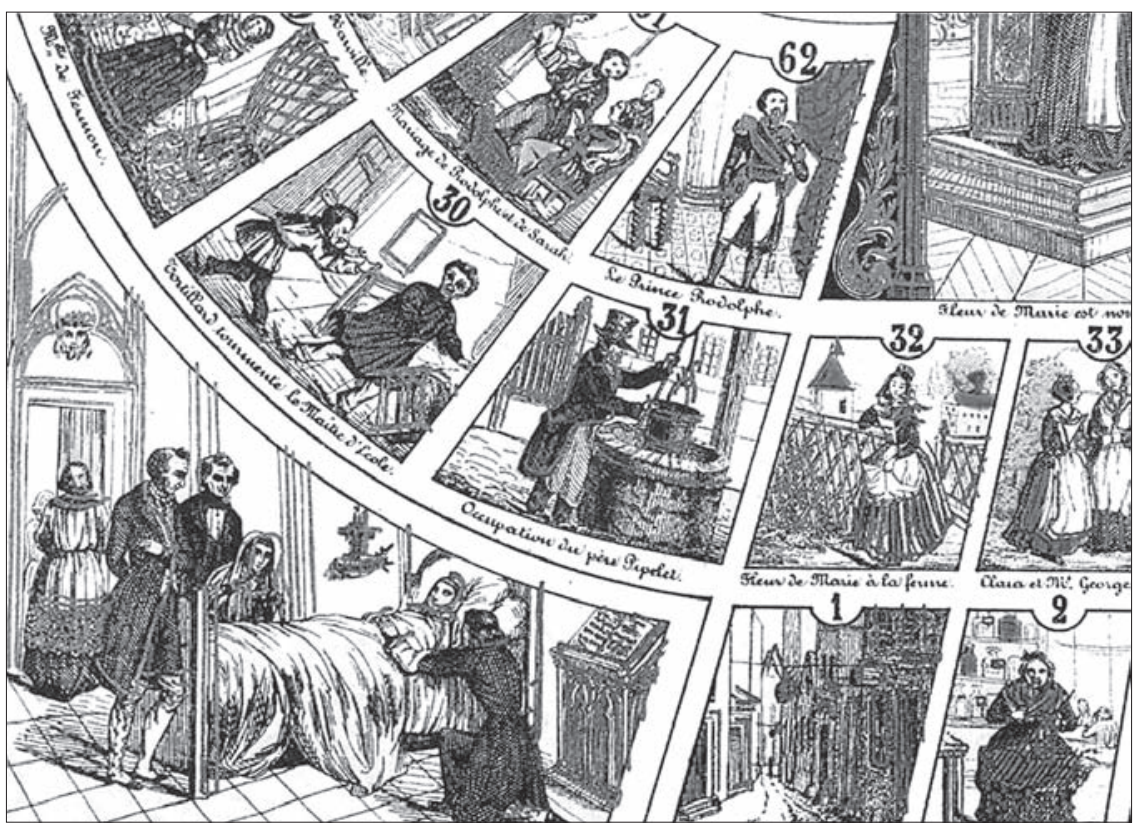

Fig. 1. Jeu de l'oie narratif: Le jeu des mystères de Paris (XIX ${ }^{\mathrm{e}}$ siècle)

Le livre d'Alain Girard et Claude Quétel, L'histoire de France racontée par le jeu de l'oie ${ }^{16}$ détaille les centaines de versions inspirées du XVII ${ }^{\mathrm{e}}$ siècle à nos jours par ce jeu plutôt simplet. Toutes ces versions ne sont certes pas narratives, et parmi les versions narratives, la plupart ne sont pas fictionnelles, comme le montre la prédilection des auteurs de jeux pour les thèmes didactiques. Faire apprendre en s'amusant (ce qui souvent veut dire endoctriner), telle est l'ambition qui nous vaut des jeux dédiés à la succession des rois de France, à la notation musicale, aux « exercices militaires de l'infanterie française », à « l'école de vérité pour les nouveaux convertis» (jeunes filles forcées d'abjurer le protestantisme après la révocation de l'édit de Nantes), à la Révolution française, aux inventions du XIX ${ }^{\mathrm{e}}$ siècle, aux colonies françaises, et j'en passe. Parmi les thèmes purement

16. Alain R. Girard, Claude Quétel, L'histoire de France racontée par le jeu de l'oie, Paris, Balland-Massin, 1982. 


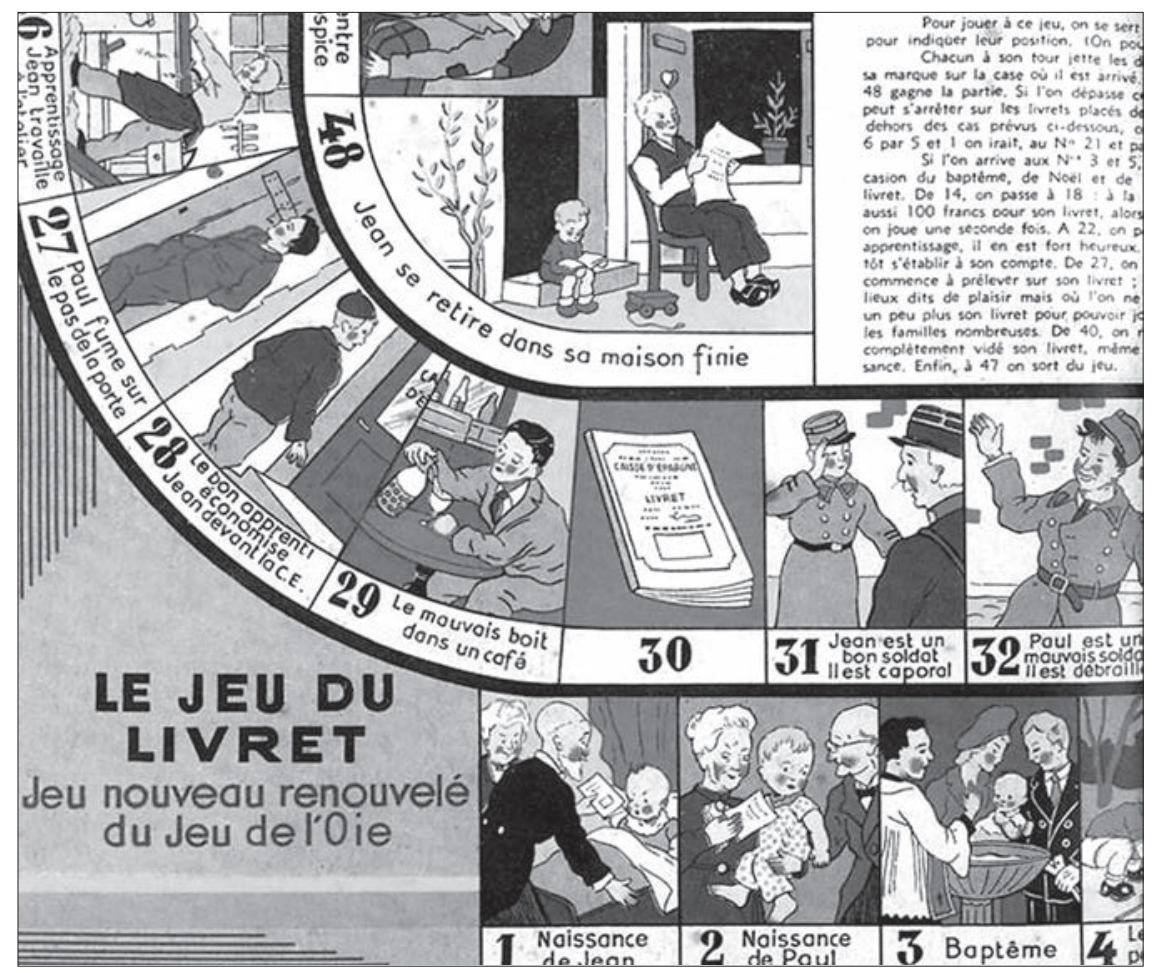

Fig. 2. Jeu de l'oie publicitaire: Le jeu du livret ( $\mathrm{xx}^{\mathrm{e}}$ siècle $)$

narratifs et fictionnels, mentionnons un jeu dédié aux fables de La Fontaine, et un autre aux Mystères de Paris (1842-1843) d'Eugène Sue. La narrativité ne se manifeste pas seulement dans les images et le texte qui remplissent les cases, mais tente parfois de s'insinuer dans la formulation des règles, comme le montre un jeu protestant basé sur la Bible ${ }^{17}$ : «Le joueur arrivé à la case 31 se lamente avec Jérémie jusqu'à ce qu'un autre joueur le rejoigne, puis il retourne à la case que ce joueur vient de quitter. » La division du labyrinthe en cases distinctes donne à certains de ces jeux l'apparence d'une bande dessinée, telle par exemple une satire de la vie militaire de l'entre-deux-guerres «à distribuer aux soldats » digne de paraître dans les pages de comics des journaux contemporains. Mais plus le jeu se rapproche de la bande dessinée, moins il semble être joué. Comme l'écrivent Girard et Quétel, « les jeux de l'oie postérieurs à la Libération semblent avoir été de plus en plus des pseudo-jeux, prétextes à une satire politique, mais

17. Exhibé au Musée de la Réformation à Genève. 


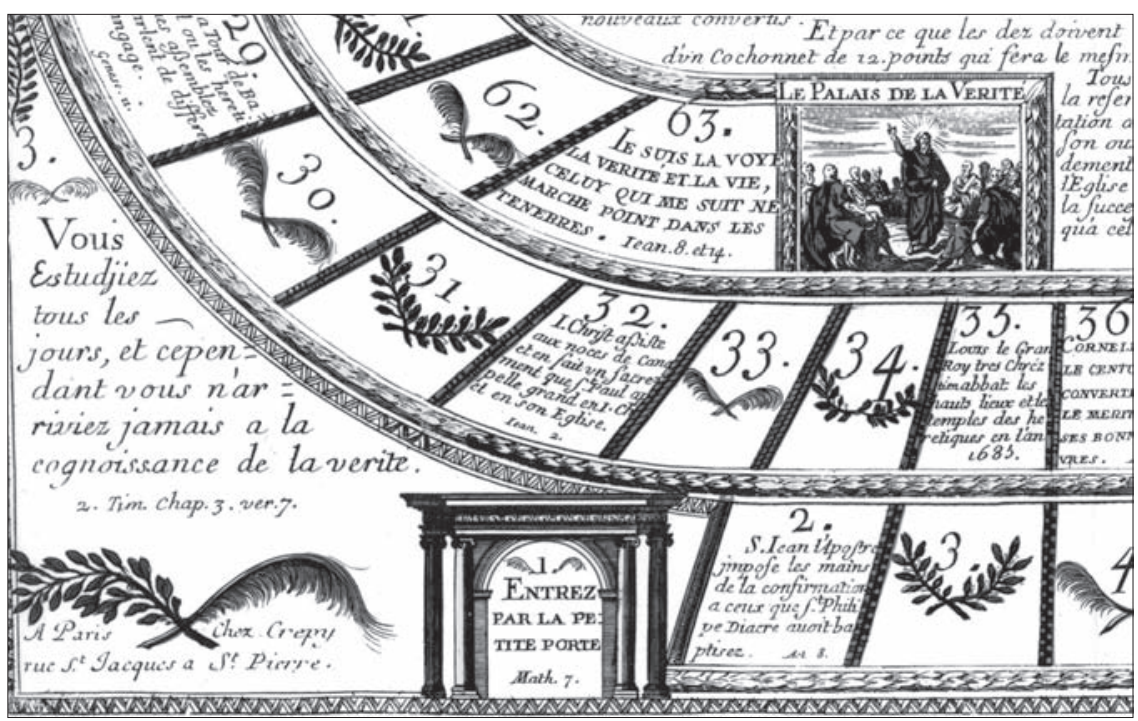

Fig. 3. L'École de la Vérité pour les Nouveaux convertis (XVII ${ }^{\mathrm{e}}$ siècle)

sur lesquels on ne jouait plus ${ }^{18} »$. C'est également le cas des nombreux jeux dédiés à la publicité ( «vins de France », « jeu du livret» distribué par une banque). Dans ces exemples, la narrativité se développe au détriment du jeu.

Le désir de jouer l'histoire semble l'emporter sur celui d'agrémenter le jeu dans le Labyrinthe de l'Arioste, une forme narrative du jeu de l'oie décrite en 1682 par le père jésuite Claude-François Ménestrier dans un ouvrage dédié à l'art du ballet de cour ${ }^{19}$. L'auteur voit dans la narrativité une manière de récupérer pour l'éducation du public ces activités généralement oiseuses et donc condamnables que sont les jeux. (On serait tenté d'associer ce terme d'oiseux au jeu de l'oie, mais il dérive en fait du latin otium, loisir.) «Il seroit à desirer ", écrit Ménestrier, «que quelqu'un entreprît de mettre l'Iliade \& l'Odyssée d'Homère, l'Eneïde de Virgile, les Métamorphoses d'Ovide, \& l'Histoire méme Grecque, Romaine, \& de divers pays en de semblables jeux, \& qu'on leur ôtat tous ces jeux d'oye, \& de Dames, qui ne servent à rien » (BA, p. 221, orthographe originale). Le Labyrinthe de l'Arioste représente l'intrigue, elle-même dédalienne, de l'Orlando Furioso (Ludovico Ariosto, 1516), poème de plus de 40,000 vers. Chaque joueur

18. Girard et Quétel, L'histoire de France, p. 147.

19. Claude-Francois Ménestrier, Des ballets anciens et modernes selon les règles du théâtre, Paris, René Guignard, Minkoff Reprints, Genève, 1972 [1682], p. 302 sq. Désormais, les références à cet ouvrage seront indiquées par le sigle «BA», suivi de la page, et placées entre parenthèses dans le corps du texte. 
adopte un pion à l'effigie d'un personnage, et le fait avancer sur un labyrinthe de 330 cases dont 89 représentent des épisodes du poème. (On ne sait pas s'il était permis de choisir un personnage du sexe opposé, comme c'est le cas dans les jeux en ligne contemporains). Isolant chacune une scène du flux du récit, les cases à figures ne permettent pas de reconstituer la logique narrative de l'intrigue. Leur rôle est d'illustrer, de rendre présent à l'imagination, plutôt que de raconter: «198. Marphise prisonniere de Bradamante. 202. Aquilante suit Marphise. 206. Astolfe délivre les prisonniers de Pinabel. 210. Marphise avec Gabrine en croupe. 213. Maison où se trouvent les choses perduës. 215 Astolfe délivre Roland» (BA, p. 313). Un joueur non familier avec l'intrigue de l'Orlando Furioso serait bien incapable de suivre l'histoire sur la base de telles données. Les cases figurées sont liées à des règles favorables ou défavorables au joueur (certaines concernant seulement les dames, d'autres réservées aux « cavaliers »), mais elles invitent aussi les joueurs à déclamer, ou plutôt à «chanter », comme l'écrit Ménestrier (BA, p. 310), les vers où l'épisode se trouve décrit. Il est permis d'imaginer que ces épisodes servaient de prétexte à des improvisations galantes ou spirituelles, chaque joueur tenant le rôle du personnage représenté par son pion. L'attrait du jeu consiste donc bien plus à revivre l'histoire qu'à progresser vers le but: «Les Cases de figures font tout le plaisir du jeu par les diverses allusions qu'elles font à divers endroits du Poëme» (BA, p. 310).

On peut se demander pourquoi, de toutes les œuvres littéraires prisées au $\mathrm{XVII}^{\mathrm{e}}$ siècle, le désir de jouer une histoire ait favorisé l'Orlando Furioso. Il me semble que le monde de l'Orlando attire les joueurs pour certaines des mêmes raisons que les mondes des jeux en ligne contemporains, tels qu'Ultima OnLine (Origin Systems/Electronic Arts, 1997) et EverQuest (Sony Interactive Studios/ Sony Online Entertainment, 1999) ${ }^{20}$. Dans les deux cas il s'agit d'un vaste monde fictionnel habité par d'innombrables personnages: une édition de l'Orlando mentionne 43 personnages principaux, nombre énorme pour une fiction littéraire, puisque le lecteur devrait être capable de suivre chacune de leur destinées individuelles. Ce nombre de personnages donne aux joueurs du Labyrinthe un ample choix de rôles. Les mondes en ligne pour leur part sont fréquentés par des milliers, si ce n'est par des millions de joueurs, quoique ce nombre soit considérément réduit par l'organisation du jeu en serveurs distincts où ne se rencontre qu'une fraction de la population totale. Ces joueurs construisent leur avatar à partir d'un répertoire de propriétés qui permet d'infinies combinaisons. Dans les

20. Sur les mondes en ligne, voir: Edward Castronova, Synthetic Worlds: The Business and Culture of Online Games, Chicago, The University of Chicago Press, 2005, et T. L. Taylor, Play Between Worlds: Exploring Online Game Culture, Cambridge, Massachusetts, The MIT Press, 2006. 
deux cas, les destinées individuelles s'entrecroisent, se nouent et se dénouent au gré des rencontres, alliances, ruptures volontaires ou séparations accidentelles. Mais alors que le lecteur de l'Orlando peut suivre toutes ces destinées, grâce à une narration à la troisième personne qui se déplace librement dans l'espace et le temps, les joueurs en ligne sont liés à la perspective de leur avatar, et ignorent ce qui se passe ailleurs que dans la région du monde représentée sur leur écran (à moins qu'ils ne rencontrent d'autres personnages qui leur racontent leurs exploits). Chevaliers errants en quête d'aventures ou chargés de mission, les personnages du poème comme ceux des jeux en ligne passent leur temps à voyager dans une géographie aussi vaste que variée. Celle de l'Orlando s'étend de l'Écosse aux Indes, et de Paris à Jérusalem, mais elle comprend aussi des lieux imaginaires, comme l'île de l'enchanteresse Alcina, et l'intrigue mène les héros de la terre à la lune. Dans les jeux en ligne, une grande partie du plaisir consiste à explorer les paysages et à visiter les divers sites d'une géographie aussi saturée de sens que les cosmologies archaïques décrites par Mircea Eliade. La distinction religieuse entre les lieux sacrés et profanes fait place ici à une architecture spatiale qui associe certains éléments du paysage à certaines possibilités d'action : dans la forêt on risque d'être attaqué par des bêtes féroces, dans les sanctuaires on jouit de l'immunité, auprès des sources on se guérit de ses blessures, et dans les villages on se livre au commerce. Les habitants des mondes en ligne, tout comme les héros de l'Orlando, peuvent se déplacer instantanément d'une région à l'autre (ce qu'on appelle dans le jargon des jeux la télétransportation) grâce à des moyens surnaturels, tels qu'enfourcher des chevaux ailés, jeter des sorts ou boire des potions magiques. La variété de la population de l'Orlando n’a rien à envier à celle de la faune des jeux en ligne: alors que ces derniers sont peuplés de chevaliers, de druides, de trolls, d'elfes et de gnomes, le poème de L'Arioste met en scène non seulement des personnages plus ou moins historiques (Roland et Charlemagne) et des types humains (chevaliers, princesses, empereurs et vilains) mais aussi des créatures empruntées à la Matière de Bretagne (l'enchanteur Merlin), à la tradition chrétienne (l'archange Saint-Michel), et plus généralement à la mythologie et au folklore (nains, sorciers, amazones, pégases, monstres marins nommés « orques », hippogriphe, et même le dieu Neptune). Le combat représente le passe-temps favori des personnages, ainsi que la manière principale d'acquérir du mérite. Presque chacun des 46 chants de l'Orlando met en scène un duel. On y joute pour délivrer des damoiselles en détresse, pour aider Charlemagne à vaincre les Sarrasins, pour conquérir une bien-aimée (ou un bien-aimé, dans le cas de l’idylle entre Bradamante et Roger). Dans les jeux en ligne, l'enjeu du combat est plus prosaïque, puisqu'il consiste à acquérir du butin que le personnage peut échanger contre de l'argent, avec lequel il peut acheter des biens, dont certains le rendent capable de s'attaquer à des adversaires plus féroces, afin de récolter du butin, que le personnage peut échanger contre 
des biens, et ainsi de suite. Par ce scénario cyclique, qui leur permet d'accumuler pouvoir, prestige et richesses, les joueurs gravissent l'échelle sociale dans la communauté du jeu.

Toutes ces similarités au niveau de la configuration de leur monde fictionnel ne devraient pourtant pas faire oublier la différence fondamentale entre le jeu du Labyrinthe de l'Arioste et les jeux en ligne. Dans le jeu de l'Arioste, la trame narrative est riche mais le répertoire d'actions est pauvre et il n'entretient pas de relations thématiques avec le récit; dans le jeu en ligne, les actions sont relativement diverses par rapport à celles du jeu de l'oie, et elles présentent un rapport direct avec l'histoire, puisque ce sont elles qui « écrivent» la destinée des personnages, mais cette histoire ne peut rivaliser en intérêt purement narratif avec le poème de l'Arioste. Dans le jeu en ligne, on peut se déplacer, s'allier à d'autres personnages, aider, informer, bavarder, combattre, se guérir de blessures, vendre, acheter, ramasser des objets, résoudre des problèmes; et c'est en cela que consiste le contenu narratif créé dynamiquement dans le temps du jeu ${ }^{21}$. Dans le labyrinthe de l'Arioste, au contraire, le contenu précède le jeu et demeure immuable de partie en partie. L'activité des joueurs se réduit à jeter les dés et à bouger leur pion (ou à la rigueur à réciter le poème), mais les personnages de l'histoire peuvent trahir, tromper, se sacrifier, se venger, protéger, être pris de rage, espérer, regretter, se sentir coupables et, surtout, aimer - toutes dimensions fondamentales de l'expérience humaine qu'il est jusqu'à présent impossible d'intégrer dans la trame narrative d'un monde en ligne ${ }^{22}$.

21. Il existe d'autres contenus narratifs dans les jeux en ligne: les histoires qui se rapportent aux divers lieux, ou que racontent les personnages «NPC » («non-playing characters ", c'est-à-dire des personnages créés par le système), les tâches ("quêtes») que donnent les «NPC» aux joueurs, et les histoires qu'échangent entre eux les joueurs au cours de leurs conversations.

22. On dira peut-être que, dans la mesure où dans les jeux en ligne les joueurs improvisent leurs propres scénarios, au lieu d'être forcés de réaliser un schéma narratif inhérent au jeu, ils peuvent accomplir des actions les uns envers les autres qui sont perçues comme trahison, vengeance, protection, ou qui suscitent le regret, la rage, ou la fictionnalité. Et d'autre part on parle beaucoup de joueurs qui tombent amoureux les uns des autres. Mais il y a d'importantes différences entre ces scénarios et celui de l'Orlando. Tout d'abord, ils concernent les relations de joueur à joueur, et non les relations entre joueurs et personnages créés par le jeu. Dans la mesure où elles résultent d'une transaction entre joueurs individuels, ces mini-intrigues ne sont accessibles qu'à leurs participants et non pas à tous les joueurs. Elles impliquent souvent une expérience hors de rôle (par exemple des joueurs qui tombent amoureux). Et finalement, si un joueur en trahit un autre ou se venge de lui dans un monde en ligne, ce scénario n'est pas une source de plaisir pour le joueur victime, alors que les trahisons et vengeances de l'Orlando font partie d'une intrigue qui peut être appréciée par tous les joueurs-lecteurs. 
La disparité entre la trame narrative et le champ d'action offert au joueur n'est pas unique aux variations du jeu de l'oie; elle se retrouve dans la plupart des jeux vidéo basés sur un monde fictionnel préexistant. Que ce monde soit celui d'Alice au pays des merveilles (Lewis Carroll, 1865), de la série Harry Potter (J. K. Rowling, 1997 à aujourd'hui), du film The Matrix (Larry et Andy Wachowski, 1999), ou de l'histoire d'horreur The Call of Cthulhu de H. P. Lovecraft (1928), les possibilités d'action correspondent à ce que l'ordinateur permet de faire le plus facilement: mouvoir son avatar, ramasser des objets, les manipuler selon la nature de l'objet (le code qui lui est associé), ou faire un choix parmi les éléments d'un menu pour susciter certains événements. Ce répertoire prédispose les jeux basés sur une histoire à devenir de simples shooters, comme c'est le cas pour American McGee's Alice (Roque Entertainment/Electronic Arts, 2000) ou Enter The Matrix (Shiny Entertainment/Atari, 2003), ou à faire de l'histoire la récompense du joueur, quand celui-ci parvient à résoudre certains problèmes plus ou moins étroitement reliés à l'intrigue (gagner un jeu de Quidditch dans Harry Potter and the Sorcerer's Stone [Argonaut Games/Electronic Arts, 2001]). Dans le premier cas, l'histoire se réduit à un décor visuel, dans le second cas, l'activité du joueur ne sert qu'à mettre en marche une narration non interactive présentée généralement par des clips filmiques. Dépourvu d'influence sur le développement de l'intrigue, ce type d'activité révèle parcimonieusement une histoire que Chris Crawford qualifie sarcastiquement de «constipée $»^{23}$.

\section{FUSION DU JEU ET DE L'HISTOIRE : UTOPIE OU POSSIBILITÉ ?}

Ce dilemme entre la jouabilité et la narrativité donne-t-il raison à Caillois? Rappelons qu'il déclarait ennemis non le récit et le jeu mais la fiction et les règles. Or, comme nous l'avons vu, il existe des jeux non réglés - la paidia - et des fictions plus ou moins narratives. Les jeux vidéo ont amplement démontré ce que les variations du jeu de l'oie suggèrent timidement: que transformer le terrain de jeu en un monde fictionnel qui procure un plaisir visuel et stimule l'imagination n'est nullement incompatible avec la compétition disciplinée du ludus. Si les règles s'accommodent mieux avec le «faire semblant» de la fiction dans les jeux vidéo que dans les jeux de société, c'est qu’implémentées par le code de l'ordinateur elles fonctionnent différemment. Dans un jeu de société comme le jeu de l'oie, les règles sont des conventions arbitraires qui définissent le jeu et que les joueurs acceptent par accord unanime. Ce pacte ludique laisse aux joueurs l'option de tricher. Mais dans un jeu vidéo, la plupart des règles sont plus proches

23. Chris Crawford, Chris Crawford on Interactive Storytelling, p. 131. 
des lois de la physique que des conventions arbitraires des jeux de société. De même qu'on ne peut pas tricher avec la gravité, on ne peut pas agir de manière contraire au code, car le code détermine de manière absolue le champ d'action du joueur. Dans un jeu d'échecs avec un partenaire humain, on pourrait déplacer son cavalier comme une tour, en espérant que l'adversaire sera distrait, mais dans une version électronique, l'ordinateur refusera tout simplement d'exécuter la manœuvre illicite ${ }^{24}$. Alors que dans un jeu ordinaire le joueur doit apprendre les règles avant de se mettre à jouer, dans les jeux vidéo il découvre la plupart d'entre elles comme un enfant qui fait ses premiers pas dans le monde. Seules les règles les plus arbitraires, comme l'association des touches du claviers à certaines possibilités de mouvement, doivent être présentées au joueur par des instructions extérieures au monde du jeu. C'est en effet la marque d'un bon jeu qu'on puisse l'apprendre en jouant dans son monde. La plupart des règles ne sont autres que les comportements et les possibilités de manipulation attachées à certains objets, et les actions du joueur se conforment automatiquement à ces règles, tout comme nos activités sérieuses dans la vie ordinaire se conforment automatiquement aux lois de la nature. Il est bien plus facile de se livrer à un acte de faire semblant quand les règles du jeu s'attachent à des objets concrets et découlent de leur nature, que quand ces règles sont arbitrairement imposées aux joueurs.

Mais s'il est désormais possible de faire d'un terrain de jeu un monde fictionnel où la compétition prend le visage de la quête, cela ne veut pas dire que le problème de la fusion du jeu et de l'histoire soit résolu. On peut évidemment dire que tout jeu se déroulant dans l'espace mimétique d'un monde et demandant au joueur de manipuler un avatar présente une dimension narrative, puisque conformément à la définition la plus courante du récit, ces jeux mettent en scène des agents qui participent à des séquences d'événements. Les jeux en ligne ne présentent pas de macrostructure narrative imposée par un auteur, mais par le fait même d'interagir avec un monde fictionnel, chaque avatar se crée une destinée personnelle. De même dans les jeux qui attribuent à l'avatar une mission spécifique, comme les shooters «historiés », les mille manières particulières dont l'avatar peut s'acquitter de sa mission constituent autant de vies, dont certaines

24. Ce qu'on appelle tricher (cheat) dans la culture des jeux n'est pas violer le code, mais tirer avantage du code d'une manière que le programmateur n'avait pas prévue. Par exemple, certains joueurs ont découvert que dans les Sims on pouvait tout de suite se procurer un million de dollars et acheter tout ce qu'on veut, au lieu de gagner ce million en travaillant au jeu pendant des semaines ou des mois. D’autres activités productrices de «cheats» consistent à créer des modules (patches) qui modifient le code, ce dont les hackers ne se privent pas, ou à donner au joueur des tuyaux qui facilitent sa tâche. 
conduisent à la victoire et d'autres à la mort. Or qu'est-ce qu'une histoire, sinon la représentation plus ou moins partielle d'une vie (ou sa présentation, dans le mode dramatique, qui est celui des jeux vidéo)? Mais si tout jeu vidéo fictionnel est une machine à fabriquer des histoires dans ce sens très large de séquence d'actions accomplies par un agent situé dans un monde, il reste un abîme à combler pour permettre à cette séquence de susciter des réactions propres à l'art narratif, telles que le suspense, la surprise, la curiositée ${ }^{5}$, la réflexion morale, politique ou philosophique ${ }^{26}$, et un attachement émotionnel aux personnages créés par le système ${ }^{27}$.

Il n'y aura de vraie réconciliation du jeu et de l'histoire que quand deux conditions seront remplies : 1. Le joueur jouera pour l'histoire, et non pour d'autres buts, tels que battre des adversaires ou améliorer son propre score. 2. L'histoire sera interactive: au lieu de découvrir une histoire préétablie, comme c'est le cas dans les médias narratifs classiques (littérature, cinéma), le joueur construira l'histoire par ses actions, et chaque fois qu'il jouera il produira une nouvelle histoire. Le développement de jeux qui remplissent ces deux conditions est actuellement l'objet d'une fébrile activité parmi les chercheurs en intelligence artificielle, mais les créateurs se heurtent à ce que Ruth Aylett et Sandy Louchart appellent le paradoxe du drame interactif: "On the one hand the author seeks control over the direction of a narrative in order to give it a satisfactory structure. On the other hand a participating user demands the autonomy to act and react without explicit authorial constraint ${ }^{28}$. » Sera-t-il un jour possible de résoudre le paradoxe, ou bien

25. De ces effets, la curiosité et la surprise sont les plus faciles à susciter dans un jeu vidéo: par exemples, les joueurs sont généralement curieux de découvrir ce que contient le monde du jeu, et la manipulation d'objets peut mener à des surprises. Mais il faut distinguer une curiosité et une surprise proprement narratives, c'est-à-dire temporelles, puisqu'elles se rapportent au tour que vont prendre les événements pour les personnages, de ces mêmes expériences quand elles concernent l'exploration spatiale d'un monde.

26. Certains créateurs de jeux indépendants essaient de remédier à cette situation. Citons tout particulièrement Gonzalo Frasca. Mais dans ses jeux Kabul Kaboom (http:// ludology.org/games/kabulkaboom.html), Madrid, ou September 12 (http://www.newsgaming.com/newsgames.htm), le message politique se déploie aux dépens de la «jouabilité », qui ne présente guère d'intérêt. En effet, il n’est possible ni de gagner ni d'améliorer son propre score.

27. Cette dernière précision est nécessaire, car les joueurs sont généralement attachés à leur avatar.

28. Sandy Louchart et Ruth Aylett, «The Emergent Narrative: Theoretical Investigation ", actes du colloque Narrative and Learning Environments Conference NILEo4, Edinburgh, Écosse, 2004, p. 2. Accessible à l'adresse: http://www.macs.hw.ac.uk/ sandy/ ENFramesetPage.htm 
faudra-t-il sacrifier le contrôle de l'auteur au profit d'une narrativité entièrement émergente, c'est-à-dire créée par le joueur en activant les possibilités d'action inhérentes aux habitants et aux objets du monde fictionnel? C'est actuellement les jeux d'émergence de type paidia, comme les Sims, qui offrent la solution la plus satisfaisante aux deux conditions mentionnées ci-dessus, bien que les histoires créées par ces jeux ne possèdent pas la clôture d'une intrigue classique. L'intérêt narratif des Sims tient dans le fait qu'au lieu d'engager le joueur dans une quête essentiellement solitaire, comme le font les shooters et même les mondes en ligne ${ }^{29}$, le jeu demande au joueur de gérer la vie de plusieurs personnages, ainsi mettant en scène ce qui constitue le thème par excellence de la narration: la complexité des réseaux de relations humaines ${ }^{30}$. Ces relations demeurent assez rudimentaires dans l'état actuel de tels systèmes, mais on peut espérer qu'elles gagneront en subtilité ainsi qu'en diversité dans des versions futures. Parmi les tentatives de réconcilier le contrôle de l'auteur avec l'initiative du joueur, de manière à créer des effets dramatiques de crise et de dénouement (chose impossible dans un système tel que les Sims), on peut mentionner Façade, le drame interactif de Michael Mateas et Andrew Stern (2005), et les recherches de Chris Crawford et Nicolas Szilas ${ }^{31}$. Il reste toutefois beaucoup à faire pour rendre de tels systèmes capables de réagir aux actions du joueur de manière non seulement cohérente et diverse, mais aussi conforme aux normes de l'esthétique narrative. Les projets de Crawford et de Szilas sont actuellement encore en voie de développement. Seul Façade, un scénario qui permet au joueur de participer par le langage à une querelle entre époux menant à la destruction de leur mariage, a jusqu'à présent produit une histoire véritablement jouable.

29. Cette observation peut sembler paradoxale vu le caractère éminemment social des jeux en ligne. Mais bien qu'il soit nécessaire de s'allier à d'autres joueurs afin d'accomplir des missions et de progresser dans le jeu, c'est le joueur individuel, et non le groupe auquel il se joint, qui gagne des points de mérite et s'élève de niveau en niveau.

30. Voir à ce sujet Marie-Laure Ryan, «Peeling the Onion: Layers of Interactivity in Narrative Digital Texts », 2005, en ligne, http://lamar.colostate.edu/ pwryan/onion.htm.

31. Pour Mateas et Stern, voir : Façade, drame interactif, téléchargeable à : http://interactivestory.net/download. Pour Crawford, voir le projet Erasmatron, décrit dans Chris Crawford on Interactive Storytelling. Pour Szilas, le projet ID Tension, décrit très techniquement dans: «A Computational Model for an Intelligent Narrator for Interactive Narratives », Report C-TRo5-03, octobre 2005, Department of Computer Science, Macquarie University, New South Wales 2109, Australie. Voir aussi la collection d'articles publiée sous la direction de Nicolas Szilas, Création de récits pour la fiction interactive: simulation et réalisation, Paris, Éditions Hermès, 2006. 
Mais même si le saint Graal de l'histoire-jeu devait être un jour conquis, il ne faut pas s'attendre à une expérience qui surpasse le plaisir que nous prenons au jeu d'échecs ou à la lecture de Proust (ou plus modestement à celle du Da Vinci Code). En combinant la participation active du jeu et l'histoire fictionnelle, on ne double pas le plaisir mais on le partage entre deux sources distinctes. Reste à découvrir dans quelle proportion doit se faire ce partage pour ne pas gâcher le tout. 